\title{
Iris Feature Extraction based on Haar Wavelet Transform
}

\author{
Zhu Wen yao ${ }^{1}$, Zhou jun ${ }^{2}$, WU Yu feng ${ }^{1}$ and Wang Ming jun ${ }^{1}$ \\ ${ }^{1}$ Institute of Technology, Lishui University, Lishui 323000, China \\ ${ }^{2}$ Logistic Engineering University, Chongqing 401331 \\ hgzhou2008@163.com
}

\begin{abstract}
To improve the accuracy of iris recognition system, we propose an efficient algorithm for iris feature extraction based on 2D Haar wavelet. Firstly, the iris image is decomposed by the $2 D$ Haar wavelet three times, and then a 375-bit iris code is obtained by quantizing all the high-frequency coefficients at third lever. Finally we use similarity degree function as matching scheme. Experimental results on CASIA iris database show that our algorithm has the encouraging correct recognition rate (CRR) which is only $99.18 \%$, accompanying with very low equal error rate (EER) $0.54 \%$.
\end{abstract}

Keywords: Iris feature extraction, 2D haar wavelet, Decomposition, Detail components demodulating

\section{Introduction}

Iris feature extraction is a process which converts the change of iris texture to comparable mathematical characterization [1], so we can distinguish individual via pattern match method between any two irises. Iris feature extraction is the key step of iris recognition which includes the iris image preprocessing, the feature extraction, and the matching process. In this paper, our feature extraction algorithm is based on the iris preprocessing step.

For the last decade, the most typical and successful iris recognition system was developed by Daugman [2-3], he used 2D Gabor filters to demodulate texture phase structure information in complex plane, and obtaining 2048-bits iris code to describe an iris. Phase information, rather than amplitude information is extracted to exclude influence from extraneous factors, such as imaging contrast, illumination and camera gain, the difference between a pair of iris code was measured by their Hamming distance. Wildes [4] decomposed the iris region to a Laplacian pyramid constructed with four different resolution levels by application of Laplacian of Gaussian filters and the filtered image is realized as iris features, then the normalised correlation and Fisher's linear discriminant is used to determine whether the input image and the model image are from the same class. Boles [5] considered the iris region as a series of onedimensional (1-D) concentric circle, they adopted the zero-crossing of 1-D dyadic wavelet transform on concentric circle to represent the feature of the iris, and iris matching was based on two dissimilarity functions. Lim [6] decomposed an iris image into four levels using 2-D Haar wavelet transform and quantized the fourth-level diagonal high-frequency coefficients and average value of the other three level diagonal high-frequency coefficients to form an 87-bit code. A modified competitive learning neural network (LVQ) was adopted for classification. Ma [7] decomposed the normalized iris region into a series of 1-D intensity signals by Dyadic wavelet 
transform and extracted local extreme of the wavelet transform results as iris features, a fast matching scheme based on exclusive OR operation was performed to compute the similarity between a pair of irises. In this paper, we propose an efficient algorithm for iris feature extraction. The whole procedure of our algorithm includes follow steps: firstly, the iris feature was extracted from the top right region in the normalized iris image after the iris preprocessing. Secondly, we demodulated the third-level highfrequency coefficients to obtain a 375-bit iris code using 2-D Harr wavelets transform. Finally, we perform matching scheme by similarity degree function.

\section{Iris Image Preprocessing}

Iris image preprocessing is composed of three steps: iris localization, noise detection, image normalization. The original eye image is composed of pupil, the sclera, and some eye surroundings (Eyelashes, eyelid, etc.) so the iris localization means the iris border should be localized followed by noise detection including detection of the influence of surroundings. In normalization stage, the iris region is unwrapped to a rectangular block with the fixed size in order to get rid of the dimensional inconsistencies caused by pupil dilation and other sources.

Iris localization is based upon the algorithm proposed in [2].Noise detection is based upon the algorithm proposed in [3], rubber sheet model devised by Daugman [2] is adopted to normalize iris image. The adopted iris image preprocessing method is not detailed here, because it is not the focus of this paper. However, it is very important for the subsequent processing. An example of the iris image preprocessing is shown in Figure 1.

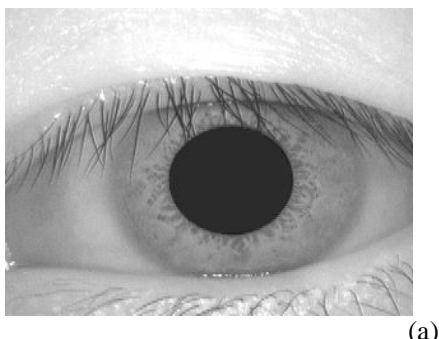

(a)

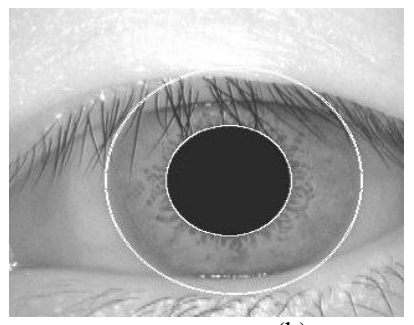

(b)

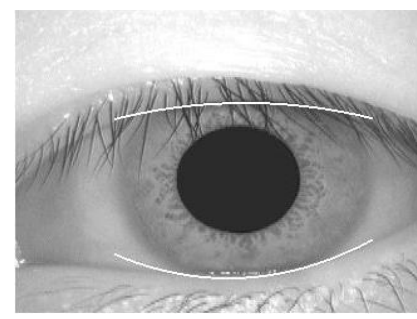

(c)

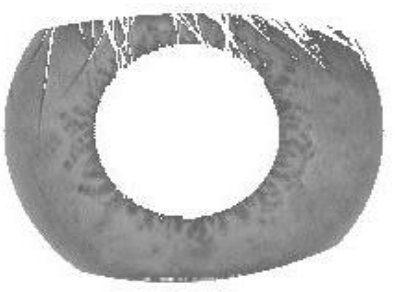

(d)

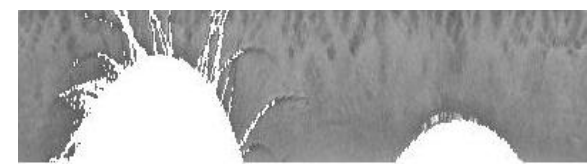

(e)

Figure 1. Iris Image Preprocessing: (a) Original Eye Image; (b) Iris Localization; (c) Eyelashes Detection; (d) Eyelid Detection; (e) Normalized Iris Image

\section{Feature Extraction and Matching}

\section{A. Image Analyses by Using 2-D Discrete Wavelet Transform}

The image is decomposed to approximate components and detail components by using 2-D scale function and 2-D wavelet function, the scale function and the wavelet function is defined as follows: 


$$
\begin{aligned}
& \varphi_{j, m, n}(x, y)=2^{j / 2} \varphi\left(2^{j} x-m, 2^{j} y-n\right) \\
& \psi_{j, m, n}^{i}(x, y)=2^{j / 2} \varphi^{i}\left(2^{j} x-m, 2^{j} y-n\right), i=\{H, V, D\}
\end{aligned}
$$

where $\psi_{j, m, n}^{i}(x, y)$ denotes wavelet function sensitive to direction. The image analyses by 2-D discrete wavelet transform is described as follows:

$$
\begin{gathered}
W_{\varphi}\left(j_{0}, m, n\right)=\frac{1}{\sqrt{M N}} \sum_{x=0}^{M-1} \sum_{y=0}^{N-1} f(x, y) \varphi_{j, m, n}(x, y) \\
W_{\psi}^{i}(j, m, n)=\frac{1}{\sqrt{M N}} \sum_{x=0}^{M-1} \sum_{y=0}^{N-1} f(x, y) \varphi_{j, m, n}^{i}(x, y), i=\{H, V, D\}
\end{gathered}
$$

$f(x, y)$ denotes image intensity function, the size of image is $M \times N$. where $N=M=2^{J}$, $j=0,1,2, \cdots, J-1, m, n=0,1,2, \cdots, 2^{j}-1 . j_{0}$ denotes the random starting scale, commonly, $j_{0}=0$. Approximate component at scale $j$ is defined by $W_{\varphi}\left(j_{0}, m, n\right)$. Three directional detail components for $j \geq j_{0}$ is defined by $W_{\psi}^{i}(j, m, n)$.

The decomposition process by 2-D discrete wavelet transform from the high scale to the low scale is shown in Figure 2. $L L$ (corresponding to $W_{\varphi}$ ) indicates approximate components. $H H, H L, L H$ (corresponding to $W_{\psi}{ }^{D}, W_{\psi}{ }^{H}$ and $W_{\psi}{ }^{V}$ ) indicates detail components. The secondlevel decomposition is expanded on $L L_{l}$ component as the above process.

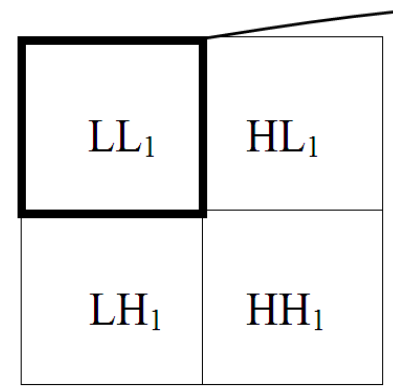

(a)

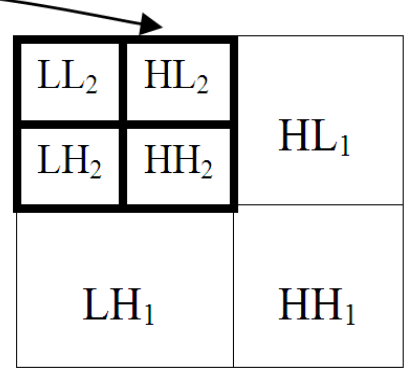

(b)

\section{Figure 2. Image Analyses by Using 2-D Discrete Wavelet Transform: (a)} Decomposition at First Level; (b) Decomposition at Second Level

\section{B. Feature Extraction and Encoding}

Figure 3(a) is an example of the normalized iris image. The $R_{1}$ region is occluded by Eyelashes and upper eyelid. The $\mathrm{R}_{2}$ region is occluded by bottom eyelid, and we find the most iris texture close to the pupil. Therefore, we extract features from $\mathrm{R}_{3}$ region. In this paper, the size of the normalized iris image is $100 \times 400$.Morever, the feature extraction region should not less than $60 \%$ of the normalized iris image in size [11].So the size of R3 region is $40 \times 200$.

We used Haar wavelet illustrated in Figure 3(c) as a basis wavelet function. We apply wavelet transform in $\mathrm{R}_{3}$ region three times, Figure $3(\mathrm{~b})$ how's the results. We obtain approximate components $\mathrm{LL}_{3}$, horizontal detail components $\mathrm{LH}=\left\{\mathrm{LH}_{1}, \mathrm{LH}_{2}, \mathrm{LH}_{3}\right\}$, vertical detail components $\mathrm{HL}=\left\{\mathrm{HL}_{1}, \mathrm{HL}_{2}, \mathrm{HL}_{3}\right\}$ and diagonal detail components $\mathrm{HH}=\left\{\mathrm{HH}_{1}, \mathrm{HH}_{2}, \mathrm{HH}_{3}\right\}$. Fig. 3(d) shows the conceptual process of the wavelet transform with Haar wavelet. 
The detail components represent the most information of iris texture. If we represented the iris feature using the detail components at the first or second lever, the feature space will be large, and leading to more cost on consumption. After large numbers of tests, we found it is appropriate to represent the iris feature using the detail components at the third lever. In fact, the iris feature is coefficients from wavelet transform, the value of coefficients is positive or negative, but positive or negative value represents entirely different comparability between the wavelet and section of the signal [8]. So we encoded iris feature as following regulation.

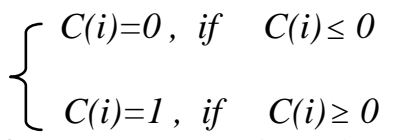

Where $\mathrm{C}$ represents iris feature space and $\mathrm{C}=\left\{\mathrm{LH}_{3}, \mathrm{HL}_{3} \mathrm{HH}_{3}\right\}$, and $\mathrm{C}(\mathrm{i})$ is the element of C. Altogether 375 binary code are computed for each iris.

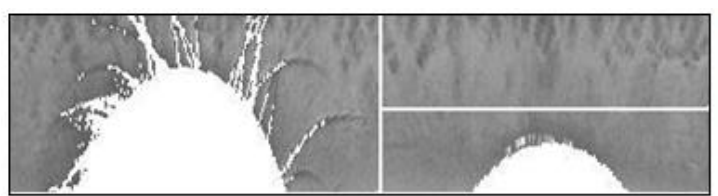

(a)

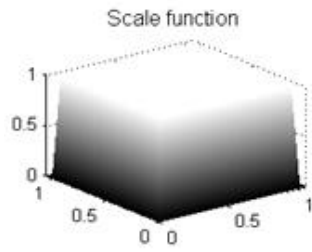

Wavelet function (V)

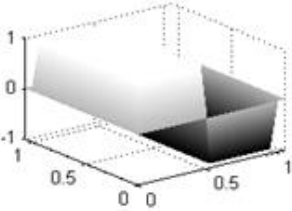

(c)

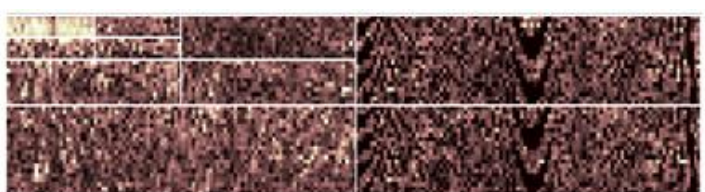

(b)

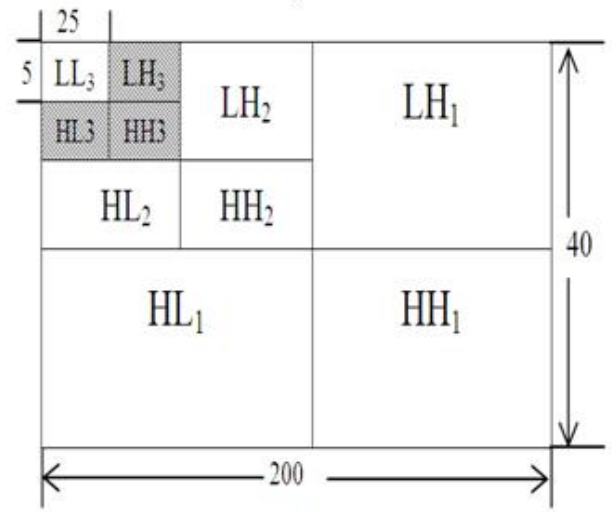

(d)

Figure 3. Sketch Map of Iris Feature Extraction: (a) Select a Feature Extraction Region;(b) Results of Wavelet Transform with 2-D Haar Wavelet ;(c) 2-D Haar Scale Function and Wavelet Function; (d) Conceptual Diagram for Organizing an iris Feature

\section{Matching}

The final stage is to determine whether two irises represented as binary codes are from the same class. We usually use HD (Hamming distance) [2] defined as Eq.6 for matching, and $\mathrm{HD}$ is in inverse proportion to similarity degree between a pair of irises.

$$
H D(A, B)=\frac{1}{N} \sum_{j=1}^{N} A_{j} \oplus B_{j}
$$

Where $\mathrm{A}$ and $\mathrm{B}$ denote different iris feature codes, $\mathrm{Aj}$ and $\mathrm{Bj}$ is corresponding bit of iris feature codes, $\mathrm{N}$ is the size of an iris feature code.

We use SD (similarity degree) for matching; the SD is defined as following:

$$
S D(A, B)=1-H D(A, B)
$$

Actually, SD is consistent with $\mathrm{HD}$, but $\mathrm{SD}$ is in direct proportion to similarity degree 
between a pair of irises, and SD is accord with thinking habit .Finally, we set a threshold T, if $\mathrm{SD}(\mathrm{A}, \mathrm{B}) \geq \mathrm{T}, \mathrm{A}$ and $\mathrm{B}$ is from the same class.

\section{EXPERIMENTAL RESULTS}

The proposed algorithm was been tested on the CASIA (ver. 1.0) database. This database contains 756 iris images with a resolution of $320 \times 280$ pixels. There are 108 iris classes with 7 different images from each class. The first image of each class was used to generate 108 enrollment templates. In verification (one-to-one matching) mode, Comparing with other iris images of the same class, the total number of intra-class comparisons is 628. In identification (one-to-many matching) mode, Comparing with the first iris image of other 107 iris classes, the total number of inter-class comparisons is 11556.

\section{A. Performance Evaluation of the Proposed Method}

The false accept rate (FAR) is the probability of accepting an imposter as an authorized subject and the false reject rate (FRR) is the probability of an authorized subject being incorrectly rejected. Total error rate (TER) is defined as follows:

$$
T E R=F A R+F R R
$$

And Total accurate rate (TAR) is defined as follows:

$$
T A R=100 \%-T E R
$$

As Table 1 shows, TAR of our algorithm is above $98 \%$ in a relatively wide threshold range.

Table 1. Performance in Different Classify Threshold

\begin{tabular}{ccccc}
\hline Threshold & FAR(\%) & FRR(\%) & TER(\%) & TAR (\%) \\
\hline 0.615 & 1.2473 & 0.0000 & 1.2473 & 98.7527 \\
0.620 & 0.5670 & 0.3700 & 0.9370 & 99.0360 \\
$\mathbf{0 . 6 2 5}$ & $\mathbf{0 . 4 5 3 6}$ & $\mathbf{0 . 3 7 0 0}$ & $\mathbf{0 . 8 2 3 6}$ & $\mathbf{9 9 . 1 7 6 4}$ \\
0.630 & 0.4536 & 1.1110 & 1.5646 & 98.4354 \\
0.635 & 0.2268 & 1.6770 & 1.9038 & 98.0962 \\
\hline
\end{tabular}

Figure 4 shows distributions of intra-class and inter-class matching similarity degree. Also it reveals that the distance between the intra-class and the inter-class distribution is large, indicating the good discriminability of the proposed algorithm. Figure 5 shows distributions of error rate including FAR and FRR. Equal error rate (EER) is the point where FAR and FRR are equal in value, and it is used to evaluate the performance of the iris recognition algorithm, the lower EER means the higher performance of the iris recognition algorithm. Our algorithm gets the very low EER which is only $0.54 \%$. 


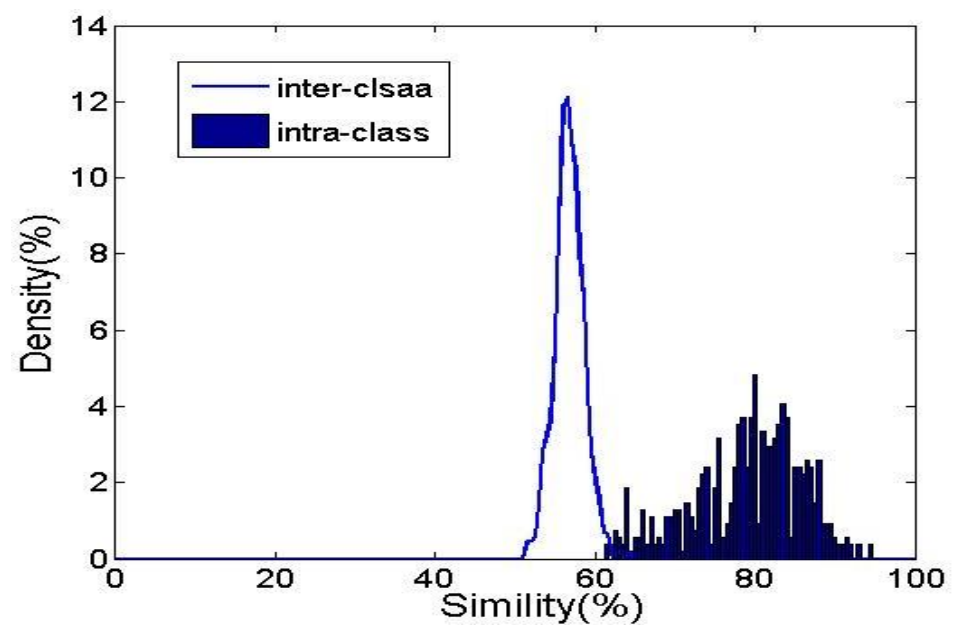

Figure 4. Distributions of Matching Similarity Degree

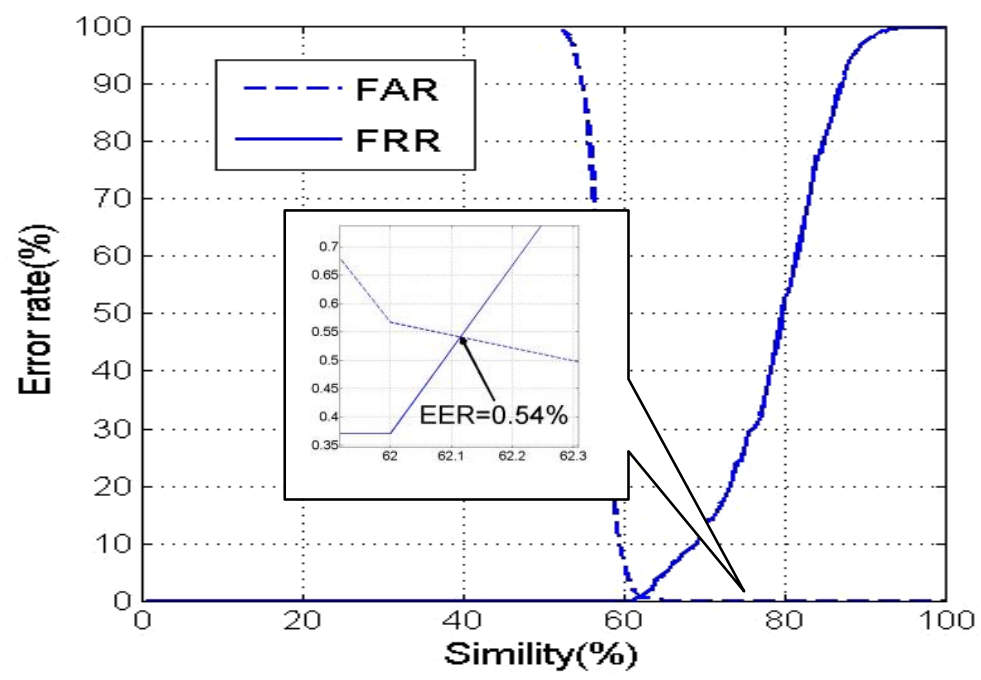

Figure 5. Curve of Error Rate Variation

\section{B. Comparison with Existing Methods}

The experiment results were compared with existing typical algorithms in two modes: verification and identification. In verification mode, the receiver operating characteristic (ROC) curve and EER are used to evaluate the performance of the proposed algorithm. The ROC curve is the track of FAR versus FRR, which measures the accuracy of matching process and shows the overall performance of an algorithm, The closer to the origin of coordinates the ROC curve is, the better the overall performance of algorithm. Figure 6 shows ROCs of our method and existing typical methods. The ROC curve of our method is smooth, indicating robust in performance. In identification mode, the algorithm is measured by correct recognition rate (CRR), the ratio of the number of samples being correctly classified to the total number of test samples. Table 2 describes the CRRS and EERS of our algorithm and existing typical algorithms, because the Wildes' algorithm only works in verification mode [9], we do not test its performance in identification mode. Table II and Figure 5 shows that Daugman's algorithm have the best performance, followed by our algorithm. Comparing with 
Boles' method and Lim's method which is based on wavelet transform, our algorithm shows better performance.

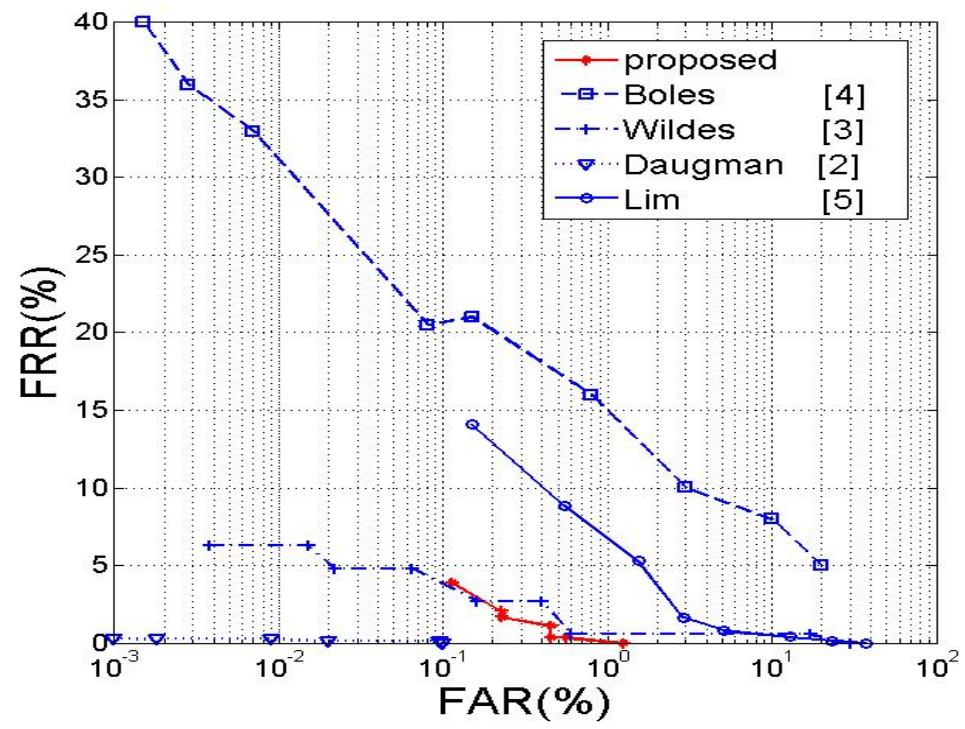

Figure 6. Comparison of ROCs

Table 2. Comparison of CRR and EER

\begin{tabular}{ccc}
\hline Methods & CRR (\%) & ERR $(\%)$ \\
\hline Daugman $^{[2]}$ & 100 & 0.08 \\
Boles $^{[4]}$ & 92.64 & 8.13 \\
Wildes & - & 1.76 \\
Lim & 98.4 & 2.3 \\
proposed & $\mathbf{9 9 . 1 8}$ & $\mathbf{0 . 5 4}$ \\
\hline
\end{tabular}

\section{Discussions}

Analyzing the experiment results shown in Figure 4(a), we observe the occlusion of eyelids and eyelashes will incur larger standard deviation of intra-class distribution. So we are developing more accurate method to detect eyelids and eyelashes. In addition, further testing on verification mode is needed, e.g., testing in CASIA (version 2.0) database. Boles' algorithm is based on 1-D wavelet, lacking consideration on correlation of 2-D iris image, so his method is easily affected by quality of iris image. Method by Lim is similar with our algorithm, but he just extracted an 87-bit iris code, comparing with our 375-bit iris code, our method is more powerful in representing the texture features, and reducing probability of false match rate. 


\section{Conclusion}

In this paper, we proposed an effective algorithm for iris feature extraction using 2D wavelet transform. Comparing with existing typical algorithm, we consider not only correlation of 2-D iris image but also the size of iris feature code. The size of our iris feature code is only $20 \%$ compared with Daugman's. Experimental results have illustrated the encouraging performance of the proposed method among typical algorithm. In order to improve performance, an investigation to eyelashes and eyelid detection should be carried out. In addition, further testing on other iris databases is needed.

\section{References}

[1] Q. Tian, "Theroy and algorithm for iris recognition", BeiJing, National Defence Industrial Press, (2010).

[2] J. Daugman, "High confidence visual recognition of persons by a test of statistical independence", IEEE Trans. Pattern Analy. Machine Intell., vol. 15, (1993) November, pp. 1148-1161.

[3] J. Daugman, "Statistical richness of visual phase information: update on recognizing persons by iris patterns", Int. J. Comput. Vis., vol. 45, no. 1, (2001), pp.25-38.

[4] R. Wildes, "Iris recognition: an emerging biometric technology", Proc. IEEE, vol. 85, (1997) September, pp. $1348-1363$.

[5] W. Boles and B. Boashash, "A human identification technique using images of the iris and wavelet transform”, IEEE Trans. Signal Processing, vol. 46, (1998) April, pp. 1185-1188.

[6] S. Lim, K. Lee, O. Byeon and T. Kim, "Efficient iris recognition through improvement of feature vector and classifier”, ETRI J., vol. 23, no. 2, (2001), pp.1-70.

[7] L. Ma, Y. Wang and T. Tan, "Iris recognition based on multichannel Gabor filtering", In Proc. 5th Asian Conf. Computer Vision, vol. I, (2002), pp. 279-283.

[8] S. Mallat, "Zero-crossings of a wavelet transform”, IEEE Trans. on Inform. Theory, vol. 37, (1992) July, pp. 1019-1033.

[9] R. Wildes, J. Asmuth, G. Green, S. Hsu, R. Kolczynski, J. Matey and S. McBride, "A machine-vision system for iris recognition”, Mach. Vis.Applic., vol. 9, (1996), pp. 1-8. 